\title{
On the Prevalence and Characteristics of MPLS Deployments in the Open Internet
}

\author{
Joel Sommers \\ Colgate University \\ jsommers@colgate.edu
}

\author{
Brian Eriksson \\ Boston University \\ eriksson@cs.bu.edu
}

\author{
Paul Barford \\ University of \\ Wisconsin-Madison and \\ Qualys, Inc. \\ pb@cs.wisc.edu
}

\begin{abstract}
Multi-Protocol Label Switching (MPLS) is a mechanism that enables service providers to specify virtual paths through IP networks. The use of MPLS in the open Internet (i.e., public end-to-end paths) has important implications for users and network neutrality since MPLS is frequently used in traffic engineering applications today. In this paper we present a longitudinal study of the prevalence and characteristics of MPLS deployments in the open Internet. We use path measurement data collected over the past 3.5 years by the CAIDA Archipelago project (Ark), which consist of over 10 billion individual traceroutes between hosts throughout the Internet. We use two different techniques for identifying MPLS paths in Ark data: direct observation via ICMP extensions that include MPLS label information, and inference using a Bayesian data fusion methodology. Our direct observation method can only identify uniform-mode tunnels, which very likely underestimates MPLS deployments. Nonetheless, our results show that the total number of tunnels observed in a given measurement period has varied widely over time with the largest deployments in tier- 1 providers. About $7 \%$ of all autonomous systems deploy MPLS and this level of deployment has been consistent over the past three years. The average length of an MPLS tunnel has decreased from 4 hops in 2008 to 3 hops in 2011, and the path length distribution is heavily skewed. About $25 \%$ of all paths in 2011 cross at least one MPLS tunnel, while $4 \%$ cross more than one. Finally, data observed in MPLS headers suggest that many ASes employ some types of traffic classification and engineering in their tunnels.
\end{abstract}

\section{Categories and Subject Descriptors}

C.2.3 [Network Operations]: Network management, Network monitoring; C.2.5 [Local and Wide-Area Networks]: Internet (e.g., TCP/IP; C. 4 [Performance of Systems]: Measurement Techniques

\section{General Terms}

Algorithms, Design, Experimentation, Measurement, Performance

Permission to make digital or hard copies of all or part of this work for personal or classroom use is granted without fee provided that copies are not made or distributed for profit or commercial advantage and that copies bear this notice and the full citation on the first page. To copy otherwise, to republish, to post on servers or to redistribute to lists, requires prior specific permission and/or a fee.

IMC'11, November 2-4, 2011, Berlin, Germany.

Copyright 2011 ACM 978-1-4503-1013-0/11/11 ...\$10.00.

\author{
Keywords \\ MPLS, traceroute, traffic engineering, tunnels
}

\section{INTRODUCTION}

In the late 1990's, the limitations and inflexibility in IP routing and forwarding coupled with the on-going quest to improve switching performance led to the development of Multiprotocol Label Switching (MPLS). Label switching 1 was envisioned and designed as a simple mechanism that would operate between layers 2 and 3 in the standard Internet protocol stack, and enable efficient lookups at each hop on a designated path. Standardization efforts in the IETF began in 1997 [14] and have resulted in an assortment of drafts and RFCs that define and specify the protocol. Today, MPLS is a standard feature in routers and is available on a wide variety of platforms from many different vendors.

Along with improved switching speeds, MPLS was quickly recognized as a highly useful protocol for traffic engineering [10]. MPLS affords the ability to configure multi-hop tunnels that supersede shortest path routes for definable traffic aggregates. It enables service providers to operate their infrastructures in a fashion that more directly addresses their business and operational requirements. Anecdotal reports suggest that MPLS is currently used by service providers in a variety of ways including facilitating traffic engineering, implementation of multi-service networks (including virtual private networks), and improving robustness [34]. Despite its appeal and implications of its broad use, to the best of our knowledge there are no studies to date that provide a systematic empirical assessment of MPLS deployments in the Internet.

In this paper, we present a longitudinal analysis of MPLS deployments in the open Internet (i.e., paths that are not part of virtual private networks). The goal of our work is to establish a broad empirical baseline for MPLS since tunnels have a direct impact on traffic behavior and measurement, and have significant implications for network neutrality, which is an increasingly active topic of conversation. We seek to answer questions such as: How many networks use MPLS? Has the use of MPLS grown over time? What are the characteristics of individual MPLS tunnels? How likely is it that my traffic will encounter an MPLS tunnel? To address these questions, we require measurements of a large set of Internet paths conducted over a period of years.

Studies of Internet-wide phenomena related to end-to-end, routerlevel path properties typically rely on active probe-based measurement tools such as traceroute for gathering data. At first glance, MPLS appears to present an insurmountable challenge to standard TTL-limited active probing methods that rely on layer 3 messaging

\footnotetext{
${ }^{1}$ Originally it was called "tag switching" $|30|$ and had roots in Ipsilon's flow management protocol [29].
} 
since MPLS is not a layer 3 protocol. However, relatively recent extensions to the ICMP protocol [12] that enable it to include the entire MPLS label stack overcome this challenge (as long as the measurement tool and target routers implement the extensions and the router is not configured to hide the tunnel). Traceroute-style path measurements that include MPLS label stacks are the starting point for our work.

We use data collected by the Ark project [22], which, since 2008 includes MPLS label stacks wherever they are in use and visible on an end-to-end path. Network operators can configure MPLS tunnels in such a way as to hide them from traceroute. Thus, our work relies on tunnels that are configured in uniform (visible) mode, which we describe below. The Ark infrastructure and methodologies have been designed to efficiently measure all routable $/ 24$ 's in the Internet. Since its inception, Ark has conducted over 10B individual traceroute measurements, and as such offers are a compelling source of data for our longitudinal study.

Our analysis of MPLS labels in Ark path measurement data reveals a broad set of characteristics about the deployment and use of the protocol. In particular, we find that the total number of tunnels observed in any measurement period has varied widely over time from a low of about $200 \mathrm{~K}$ in ' 09 to a recent high of around $350 \mathrm{~K}$. Curiously, we find that the variability of tunnel deployments correlates closely with key economic indicators. Roughly $7 \%$ of all autonomous systems use MPLS on some subset of their paths with the largest deployments in tier-1 providers. This level of deployment has been relatively stable over the past three years. We also find that the average length of an MPLS tunnel has decreased from 4 hops in 2008 to 3 hops in 2011. The tunnel length distribution is heavily skewed with over $90 \%$ of tunnels at 7 hops or less, however some tunnels extend beyond 15 hops. Approximately $25 \%$ of all paths in 2011 cross at least one MPLS tunnel, while 4\% cross more than one, and data observed in MPLS headers suggest that many ASes employ some kinds of traffic classification and engineering in their tunnels.

While label-based analysis enables a compelling characterization of MPLS path properties, it precludes analysis of other path measurement data archives (e.g., Skitter [13]) that do not include labels. Analysis of data sets that lack the ICMP extension information could expand our perspective and enable a more comprehensive longitudinal analysis. To address this problem we develop an MPLS tunnel identification method that is based on analysis of round trip time measurements instead of labels. The observation is that with some MPLS configurations, when a packet's TTL expires, the ICMP time exceeded message will be encapsulated in an MPLS header and forwarded to the end of the tunnel prior to being sent back to the source host. This observation is the basis for a Bayesian inference method that we show to be effective for identifying MPLS tunnels. Applying this method to additional data sets to gain a broader view of MPLS deployments over time is a goal for future work.

A summary of the key findings our work are as follows. First, the increasing trend in deployments over the past three years and the wide use by tier-1 providers means that it is increasingly likely that packets will encounter an MPLS tunnel. Second, tunnels are likely to span the entire edge-to-edge distance of a transit provider, with typical transit times on the order of $10 \mathrm{~s}$ of milliseconds. Third, our examination of the use of traffic classifiers indicates that while multiple classes are not uncommon, the diversity of classes has not changed over the past three years.

The remainder of this paper is organized as follows. In Section 2 we provide an overview of MPLS, how MPLS interacts with traceroute, and our label-based method for identifying MPLS hops on an end-to-end path. In Section 3 , we provide details on the Ark data set and report the details of our label-based MPLS deployment study. In Section 4 we describe our inference-based method for identifying MPLS paths and report our findings on applying this method to the Ark data. We summarize our work and describe next steps in our study in Section 6

\section{MPLS AND TRACEROUTE}

MPLS is typically thought of as a protocol that exists between layers 2 and 3 of the Internet protocol stack. Fundamentally, it is path-based encapsulation and forwarding protocol that adds a 32-bit header to packets as they enter an MPLS tunnel, or labelswitched path (LSP). We use the terms tunnel and LSP interchangeably in this paper. The MPLS header consists of a 20-bit label, 8-bit time-to-live (TTL) field, 3-bit traffic class field, and a 1-bit end of label stack field.

Specific labels are applied to packets based on forwarding equivalence class (FEC), which is a generalization of longest-prefix match. As packets traverse an MPLS tunnel, forwarding decisions are made based on exact matching of the MPLS label. Labels are only locally significant between a pair of routers, so as packets traverse a tunnel, labels are swapped (overwritten) prior to forwarding. Hierarchies of tunnels can also be created (i.e., an alreadytunneled packet can enter a new tunnel); stacks of MPLS headers can be used for this purpose.

MPLS tunnels must be configured (and labels distributed) on each label switch router (LSR) along a designated path (series of LSRs), and supersede layer 3 routes. The flexibility to define paths through a network not simply based on shortest paths makes MPLS highly attractive for traffic engineering tasks. Details on MPLS can be found in [31], and several other IETF RFCs.

Our work relies on recent modifications to routers and traceroute programs that implement extensions to ICMP specified in RFC 4950. These extensions permit the inclusion of the entire MPLS label stack (i.e., all MPLS header information) in the ICMP message that is returned to a source host [12]. Thus, it is this information included in ICMP time exceeded messages that are generated as part of the traceroute process that allows us to positively identify a router as participating in an MPLS tunnel.

There are two basic ways in which the IP time-to-live (TTL) field is processed in the presence of an MPLS tunnel. These are referred to as uniform mode and pipe mode [9], and they determine whether an MPLS tunnel is visible to a public user of traceroute or not Figure 1 illustrates these two modes of operation.

In pipe mode, the MPLS tunnel is not exposed to a traceroute user. At LSR B in Figure 1 the IP TTL is decremented by one and inspected upon router ingress. After that, the packet is encapsulated in an MPLS header and forwarded to the next LSR along the path. The MPLS header that is constructed at the first LSR is initialized with a prespecified TTL value, typically 255 . Upon egress from a pipe mode tunnel, the MPLS header is removed and the TTL value in the IP header is unchanged. Thus, the MPLS TTL field has no relationship to the IP TTL in a pipe-mode tunnel, and the IP TTL is only decremented by one regardless of the number of LSRs that switch the packet through the tunnel.

Note that with the pipe model, the first router of an MPLS tunnel is visible, but the ICMP time exceeded response generated from the

\footnotetext{
${ }^{2}$ Note that the uniform and pipe modes more commonly refer to tunneling models to support differentiated services in MPLS networks [19]. We use these terms similar to the way in which they are used to describe TTL processing in MPLS networks in RFC 3443 [9].
} 
Pipe Mode (hidden to traceroute)

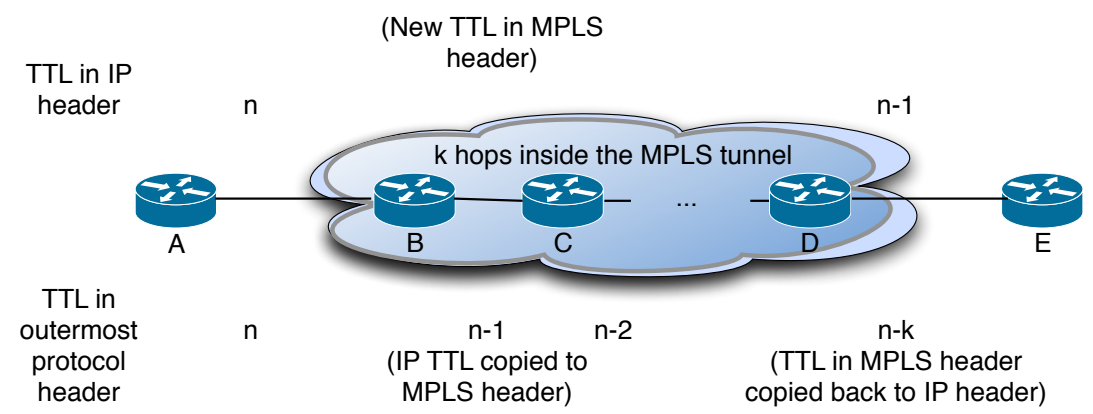

Uniform Mode (visible to traceroute)

Figure 1: Two modes of IP header TTL processing in the presence of an MPLS tunnel: pipe and uniform.

router (if the IP TTL is decremented to zero) does not indicate that the packet expired at the edge of a tunnel, since it has not actually entered one yet. Thus, there is no indication to a traceroute user that an MPLS tunnel is traversed, and only the ingress router is visible at all. (Note that penultimate hop-popping (PHP) does not affect this picture from the standpoint of traceroute [9]. We discuss PHP further in $\S 3.3$ ) The upshot is that identifying pipe mode tunnels with end-to-end measurements remains a significant measurement challenge and one that we do not address in this paper.

In uniform mode, the LSRs along an MPLS tunnel are visible to a traceroute user. At the first LSR (B in the figure), the IP TTL is decremented by one and inspected upon router ingress, just as in the pipe model. Upon encapsulation in an MPLS header, however, the IP TTL is copied into the MPLS header. At each LSR along the tunnel, the TTL in the MPLS header is decremented by one. Upon egress from a uniform mode tunnel, the MPLS TTL field is copied back to the IP header. If the TTL falls to zero at any router along the tunnel, an ICMP time exceeded message will be generated and sent back to the source of the original packet. Thus, all routers in the tunnel are visible to a user of traceroute. Importantly, only if the router implements MPLS extensions for ICMP [12] will a traceroute user be able to clearly identify the fact that the packet's TTL expired while inside an MPLS tunnel.

Interestingly, uniform mode is the default mode of operation for Cisco and Juniper routers (among others) [4. 5]. This behavior is likely due to language in the MPLS architectural RFC (3031) that specifies that the total number of hops through a tunnel SHOULD be reflected in the IP TTL when the packet emerges from the tunnel. Also, it is important to note that pipe and uniform mode TTL processing can be configured on a per-LSP basis. Even nested tunnels can have different visibility characteristics (i.e., while the innermost tunnel may be visible via traceroute, a nested tunnel may not be). From a practical perspective, this means that network operators must explicitly configure routers not to propagate the IP TTL to the MPLS header, thus hiding tunnels from the public's eye.

Another important impact that MPLS tunnels can have is on delay measurements of individual traceroute packets. When the TTL of a packet is decremented to zero inside an MPLS tunnel (in uniform mode, since that is the only way that an outsider can observe an MPLS tunnel), the ICMP time exceeded message may need to be re-encapsulated in an MPLS header and forwarded to the end of the tunnel [8]. If the LSR at which the ICMP packet is generated does not have sufficient information (e.g., routes imported via BGP), the packet will be re-encapsulated and traverse the entire length of the tunnel. At the end of the tunnel, the ICMP message can be routed back to the source host. The result is that the traceroute output will show roughly equivalent delay for the series of hops along the MPLS tunnel. In that case, we would measure the latency across the tunnel to be approximately zero. This observation is the starting point for our tunnel inference method described in Section 4

While we focus on measurement of MPLS tunnels from outside a service provider's network, internal network operators can use other mechanisms for measuring and troubleshooting MPLS LSPs. For example RFC 4379 describes MPLS-based versions of ping and traceroute for this purpose [24].

\section{ARK DATA ANALYSIS}

In this section we present our analysis of MPLS tunnels and their characteristics as observed in the CAIDA IPv4 Routed /24 AS Links Dataset [22]. Our focus in this section is on characteristics of MPLS tunnels that can be directly observed through ICMP extension information.

\subsection{Data and Limitations}

Since we do not have insight into the ways in which labels have been assigned, or generally how MPLS has been configured in a given provider network, we use a pragmatic definition of tunnel in our data analysis. Our goal in the present work is not to try to identify how different MPLS tunnels have been configured, but rather to report and analyze their observed characteristics. Our working definition of tunnel is any consecutive series of label-switched routers within the same autonomous system ${ }^{3}$ As described in Section 2 we can identify LSRs by the presence of MPLS extension information in an ICMP time exceeded message from a router. Note that with this definition, the length of a tunnel refers only to the innermost label switched path (i.e., the length of the tunnel identified by the sequence of labels at the bottom of the label stack) and does not consider nested LSPs. (We comment on nested tunnels and nested tunnel lengths below.) Note also that within an AS, we may see some number of layer 3 (IP) hops before entering a tunnel. We

\footnotetext{
${ }^{3}$ In our analysis, we detected no instances of a tunnel that spanned multiple ASes.
} 
may also see some number of layer 3 hops after exiting a tunnel, as we discuss below.

The data that we use are collected as part of the Archipelago (Ark) project, and include traceroute measurements to all routed /24 prefixes in the IPv4 Internet, initiated from a set of widely distributed probing hosts. The probing tool used in Ark is Scamper [26], and the specific traceroute method used is ICMP Paris [28]. The Ark project was initiated in 2007, and data are available starting from September of that year. However, the version of Scamper used at that time did not support the ICMP extensions for MPLS. Support for those extensions was introduced into Scamper in early 2008, and a version of Scamper with support for ICMP extensions for MPLS was rolled out to Ark in mid-May of 20084 21 27]. For that reason, we restrict our analysis in this section to the Ark data from June 2008 to August 2011. Lastly, rather than analyzing all the available Ark data from June 2008 onward, we selected the first full set of data available for each month resulting a corpus of over $250 \mathrm{M}$ individual traceroute measurements. Investigating MPLS deployment dynamics at finer levels of granularity is a topic for future study. In our analysis below, the scope of our characterizations is bounded by the Ark measurement data, and placement of probing nodes. However, the Ark project has gone to great lengths to be comprehensive and we believe it provide an important and representative view of Internet structure.

Along with the Ark data, we used the CAIDA IPv4 prefix to autonomous system (AS) mapping data, which is based on analysis of Routeviews data [1]. We also use UCLA Cyclops data to classify a given AS as a tier 1, large ISP, small ISP, or stub network [2]. We use these data to perform AS-specific analyses, and to identify the number and characteristics of ASes that appear to be employing MPLS for traffic management. Since the prefix-to-AS mapping data are based on a measured perspective of Internet routing activity, there are imperfections and complications. In our analyses, we omit any traceroute paths that have IP addresses for which we do not know the AS number, and any IP addresses that have multiple ASes identified in the prefix-to-AS mapping. We do this to simplify our analyses of specific ASes. We note that the overall amount of data discarded is small (under 1\%), thus we do not believe this materially affects our overall results.

For many analyses in this section, we wanted to identify the set of unique tunnels within an AS. We found that either using the sequence of IP addresses of the LSRs, or using the sequence of MPLS labels at the bottom of the label stack gave virtually equivalent results (differences were under $0.01 \%$ of the total number of observed tunnels).

Finally, as we discuss above, our analysis relies on the presence of ICMP extension information to positively identify MPLS LSRs. Thus, our analysis is limited to MPLS tunnels that have been configured in uniform mode. While we are certain that there are tunnels configured in pipe mode that evade our analysis, we have no way to presently quantify or characterize these tunnels. Indeed, there are ISP-published maps of MPLS networks for which we do not see any (or exceedingly few) LSRs in our data (e.g., see Sprint's [6] network map and other maps available at the Internet Topology Zoo project [7]). Moreover, we have no visibility into any MPLS virtual private networks since our measurements are taken from hosts attached to the public Internet.

\footnotetext{
${ }^{4}$ The roll-out of a new version of Scamper corresponded to the passing of the Internet Measurement Conference paper deadline that year.
}

\subsection{MPLS Prevalence}

We begin by examining the prevalence of MPLS tunnels, and how many ASes appear to use MPLS. We turn first to the total number of ASes that we observe to use MPLS. In the top plot of Figure 2, we see this number has remained fairly steady over the past 3 years, at around $7 \%$.

The bottom plot of Figure 2 shows the fraction of ASes, classified by AS type, that are empirically observed to employ MPLS. We use the UCLA Cyclops data to perform the AS classification [2]. (Note that these classification data are only available starting in October 2008.) We observe that nearly all tier 1 providers use MPLS (in October 2008 there were 8 tier 1 providers, and in August 2011 there were 11). We observe a lesser percentage of large ISPs (50 $55 \%$ ), an even smaller percentage of small ISPs (25-30\%), and few stub networks (around 5\%) to use MPLS. Because there are so many stub networks (13817 in August 2011), the overall percentage of ASes using MPLS is rather low (again, around 7\%).
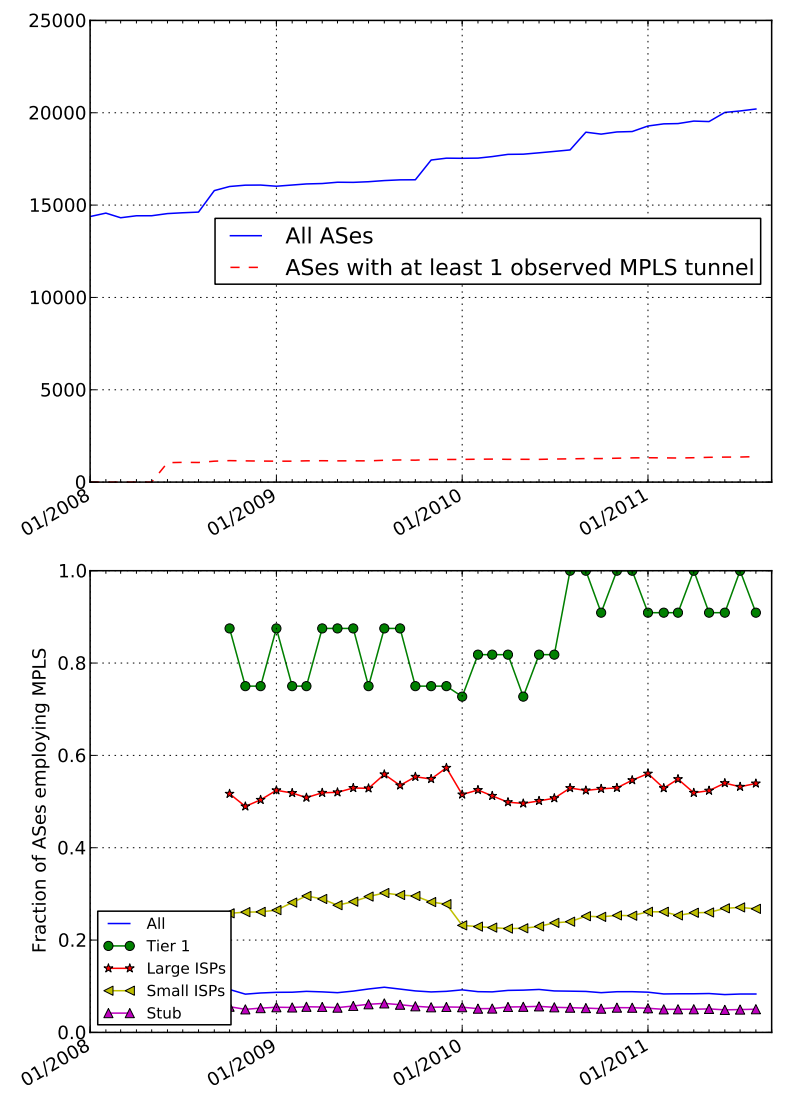

Figure 2: Comparison of the total number of ASes seen in our Ark dataset versus the number of ASes for which at least 1 MPLS tunnel is observed (top), and the fraction of ASes by AS type that employ MPLS (bottom).

Figure 3 plots the total number of unique tunnels observed in a given measurement period over the past three years. The figure shows a significant dip in the total number of tunnels beginning in '08, a rebound in mid '09 and a steady increase to the current peak of nearly $350 \mathrm{~K}$ tunnels. For comparison, we also plot the Dow Jones Industrial Average in the figure ${ }^{5}$ Interestingly, the total number of MPLS tunnels over time appears to indirectly track this eco-

${ }_{5}^{5}$ Data obtained from http://finance.yahoo.com/. 
nomic indicator (and other, similar indicators such as the S\&P 500, not shown). So, while it is apparent from Figure 2 that the sheer number of ASes using MPLS is independent of economic activity, the deployment of tunnels within these ASes roughly correlates with economic conditions. Our conjecture is that this phenomenon reflects merger and acquisition activity within the telecom industry. We have engaged ISPs to better understand the underlying reasons behind our observations, and we hope to report on our findings in the future.

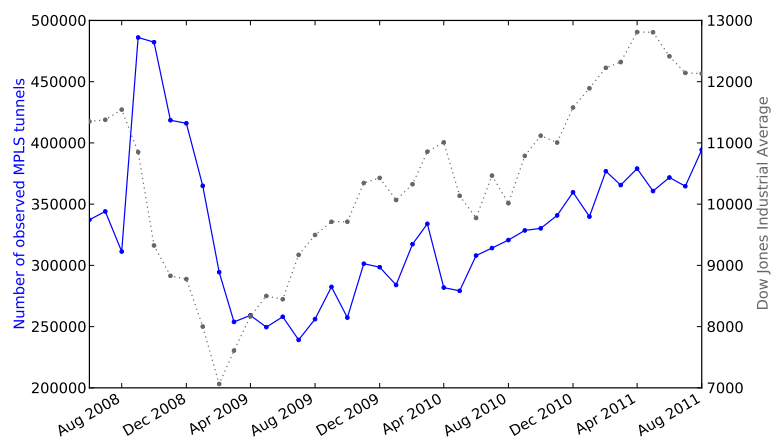

Figure 3: The total number of visible MPLS tunnels over time (solid line). The Dow Jones Industrial Average is plotted (dotted line) for reference over the same time period.

Figure 4 shows the number of unique tunnels deployed by a small set of ASes over the past three years. First, we see a sharp drop in the number of tunnels deployed by AS7018. This drop mirrors the drop in the total number of tunnels seen in Figure 3 We see from the figure that other ASes show some variability over time in the number of deployed tunnels. For example, AS1273 emerges in early 2010 from very few (nearly zero) observed tunnels. On the other hand, the number of tunnels observed from AS3320 drops to zero in mid-2010. We conjecture that in addition to economic factors, there are likely AS policy decisions (e.g., to change tunnels to "invisible" pipe mode, or to reveal previously hidden tunnels) that play a role in the observed variability.

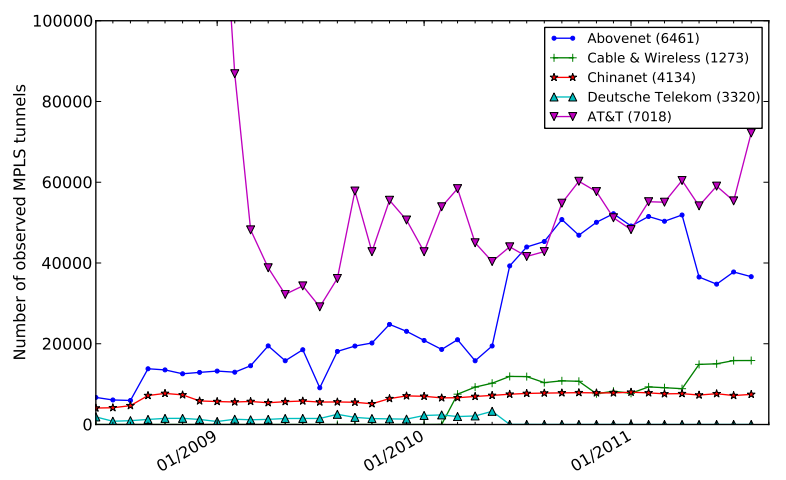

Figure 4: Number of observed MPLS tunnels over time across selected ASes.

Figure 5 provides another perspective on the number of deployed tunnels per AS. The figure shows empirical cumulative distribution functions of the number of observed tunnels per AS for six-month time intervals over the measurement period. We see that $20 \%$ of all ASes have fewer than 10 tunnels. We also see that $80 \%$ of ASes are observed to have under 200 tunnels, and that about $10 \%$ of ASes have at least 1000 tunnels.

Table 1 identifies the numbers and names of the top 10 ASes in terms of the number of observed MPLS tunnels. The table shows the top 10 ASes for six month periods starting from mid-2008. These results support the intuition and evidence in the bottom plot of Figure 2 that larger and more complex infrastructures are more likely to use MPLS tunnels.

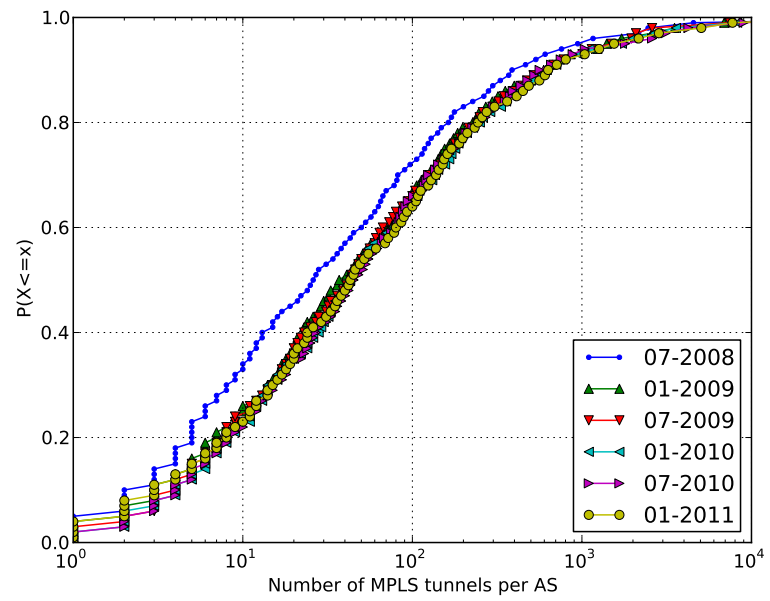

Figure 5: Empirical cumulative distribution functions of the number of visible MPLS tunnels per AS. CDFs are plotted for six month periods starting $6 / 2008$. (Note that the $x$-axis is on $\log$ scale.)

Lastly, we examine the fraction of paths probed by Ark that include one or more MPLS tunnels. Figure 6 shows the fraction of probing paths that cross at least 1 MPLS tunnel, and the fraction of paths that cross exactly 1,2 , or 3 tunnels. Interestingly, although only about $7 \%$ of all ASes are observed to use MPLS, about $25 \%$ of all probing paths crossed at least 1 tunnel in the most recent measurement data. Approximately $4 \%$ of all paths cross more than one MPLS tunnel; this observation holds across the three years. We can see that the increase in likelihood of crossing at least 1 MPLS tunnel from about 20\% in June 2008 to about 25\% in August 2011 is primarily due to higher occurrences of encountering a single tunnel on a path. This result is consistent with the fact that larger networks are more likely to appear on end-to-end paths and are more likely to deploy MPLS.

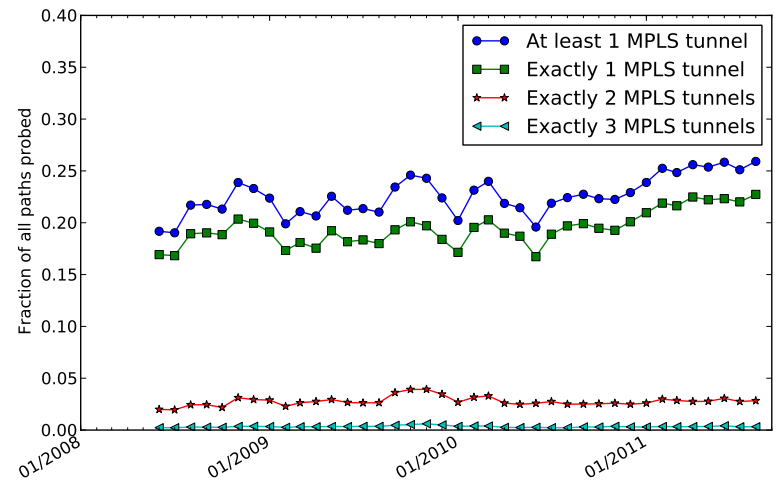

Figure 6: Fraction of paths with some MPLS. 
Table 1: MPLS heavyweights: the top 10 ASes in terms of number of observed MPLS tunnels.

\begin{tabular}{l|llllll} 
Rank & $\mathbf{7 - 1 2 / 2 0 0 8}$ & $\mathbf{1 - 6 / 2 0 0 9}$ & $\mathbf{7 - 1 2 / 2 0 0 9}$ & $\mathbf{1 - 6 / 2 0 1 0}$ & $\mathbf{7 - 1 2 / 2 0 1 0}$ & $\mathbf{1 - 6 / 2 0 1 1}$ \\
\hline 1 & 7018 AT\&T & 7018 AT\&T & 7018 AT\&T & 7018 AT\&T & 7018 AT\&T & 7018 AT\&T \\
2 & 6389 Bellsouth & 6389 Bellsouth & 6461 Abovenet & 6461 Abovenet & 6461 Abovenet & 6461 Abovenet \\
3 & 6461 Abovenet & 6461 Abovenet & 6389 Bellsouth & 19262 Verizon & 4837 China-169 & 6830 UPC \\
4 & 6453 Tata & 3269 Telecom Italia & 3269 Telecom Italia & 6389 Bellsouth & 6453 Tata & 6453 Tata \\
5 & 3292 TDC & 6453 Tata & 19262 Verizon & 6453 Tata & 1273 CW & 174 Cogent \\
6 & 4134 Chinanet & 19262 Verizon & 6453 Tata & 4837 China-169 & 6830 UPC & 1273 CW \\
7 & 4230 Embratel & 4230 Embratel & 4230 Embratel & 4230 Embratel & 4134 Chinanet & 4837 China-169 \\
8 & 19262 Verizon & 4134 Chinanet & 4837 China-169 & 4134 Chinanet & 19262 Verizon & 4134 Chinanet \\
9 & 5462 Virgin & 4837 China-169 & 4134 Chinanet & 6774 Belgacom & 6774 Belgacom & 4230 Embratel \\
10 & 4837 China-169 & 2914 NTT & 2914 NTT & 1273 CW & 4230 Embratel & 10318 Cablevision SA
\end{tabular}

Surprisingly, there were some probing paths that crossed 7 MPLS tunnels, which was the maximum we observed. Also surprisingly, within a single AS we observed multiple, separate tunnels in a single probing path. That is, from the traceroute output, we observed a series of MPLS LSR hops within an AS, followed by one or more "normal" IP hops, followed by another series of MPLS LSR hops. The frequency of occurrence of either many tunnels $(>4)$ on a single path, or multiple tunnels within a single AS was quite rare (about $0.3 \%$ of all paths in May 2011).

\subsection{MPLS Tunnel Characteristics}

We now examine characteristics of observed MPLS tunnels, both globally and within different ASes.

We first examine first-order path length statistics in ASes. We consider three segments of a path through an AS: IP hops before a tunnel is entered, hops within a tunnel, and IP hops after a tunnel, prior to exiting the AS. For this analysis, we omitted any paths through an AS that contained multiple separate tunnels. Figure 7 shows the average number of pre-tunnel, post-tunnel, and in-tunnel hops over the past three years. We see that the average length of an MPLS tunnel has decreased over the measurement period from just over 4 hops, to around 3 hops. This change likely is due to changes in operational policy or underlying infrastructure. We also see that the average number of pre- and post-tunnel hops has remained roughly constant over the past three years, at around 1 hop and 1.8 hops, respectively.

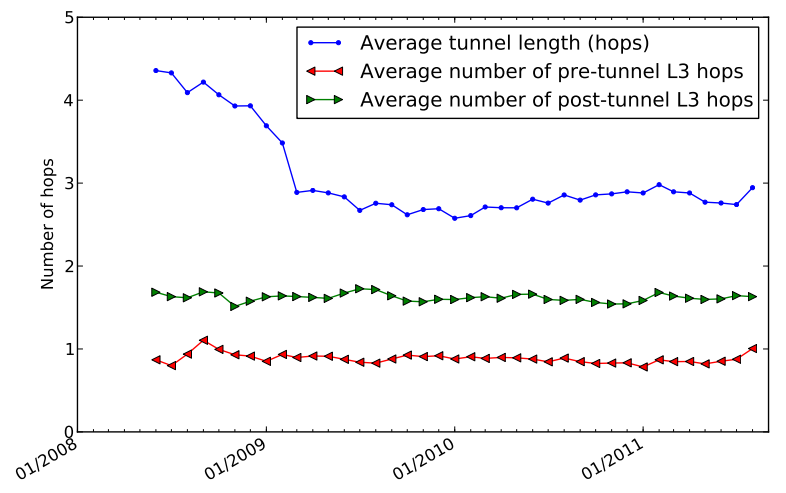

Figure 7: Average number of hops before, inside, and after MPLS tunnels for all ASes.

Figure 8 plots average tunnel lengths over time for 4 different ASes. We observe a variety of behaviors. While the average length of a tunnel in AS7018 has decreased by around 1 hop over the past three years, the average length of a tunnel in AS6461 has increased by around 2 hops. The average length of a tunnel in AS4230 has increased, but is quite short, and the plot for AS3320 shows that tunnels in that AS are currently an average of about 1 hop long.

An interesting phenomenon suggested by Figure 8 is that of very short 1-hop MPLS tunnels. In Figure 9, we show the fraction of tunnels over time that consist of one hop, for a set of ASes and globally across all tunnels. We again see a variety of behaviors. While most ASes have very few tunnels of just 1 hop, the majority of tunnels in AS4134 consist of only one hop, and tunnel lengths in AS3320 have decreased to close to a single hop.

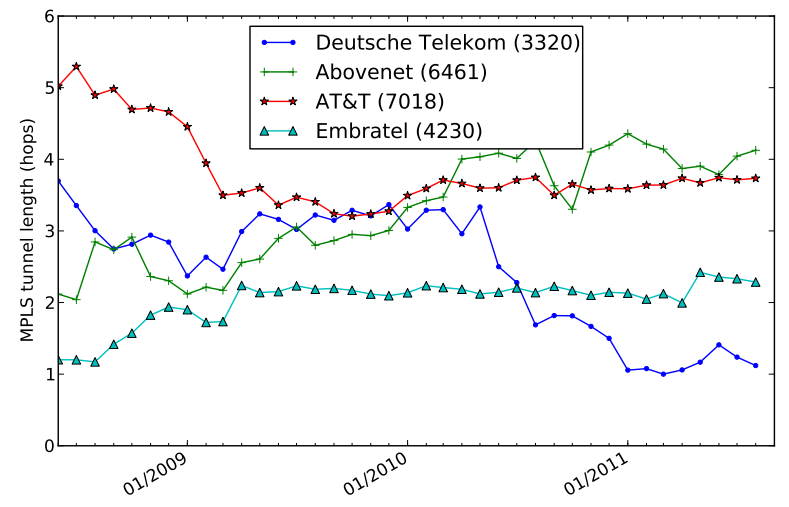

Figure 8: Average MPLS tunnel lengths for selected ASes over time.

While there are certainly operational policy decisions and other configuration factors which may lead to the observation of 1hop tunnels (e.g., there may be nested tunnels that are configured in pipe mode, making some segment of the tunnel invisible to traceroute), one simple reason we might see such short tunnels is the following. Consider the network in Figure 1] and assume that there are only three LSRs in the tunnel (B, C, and D). When a packet enters the tunnel at LSR B, it will be encapsulated in an MPLS header and forwarded to C. $\mathrm{C}$ might then do a label switch and forward it to D. At that point, the label is popped and the IP packet emerges from the MPLS tunnel.

In traceroute output, router B would appear to be a "normal" layer 3 hop since the packet has not yet been encapsulated, and routers $\mathrm{C}$ and $\mathrm{D}$ would appear to be MPLS routers. Thus, we would observe a tunnel of length 2 .

Consider, however, if the ISP has configured routers to do penultimate hop-popping (PHP), which is basically an optimization to avoid encapsulating a packet that has only one more hop in a tunnel [31], and to reduce label stack popping load on a tunnel egress router. In this case, we would only observe router $\mathrm{C}$ to employ MPLS; D would appear as a "normal" layer 3 hop. Given our definition of MPLS tunnel, we consider this to be a one-hop tunnel, 


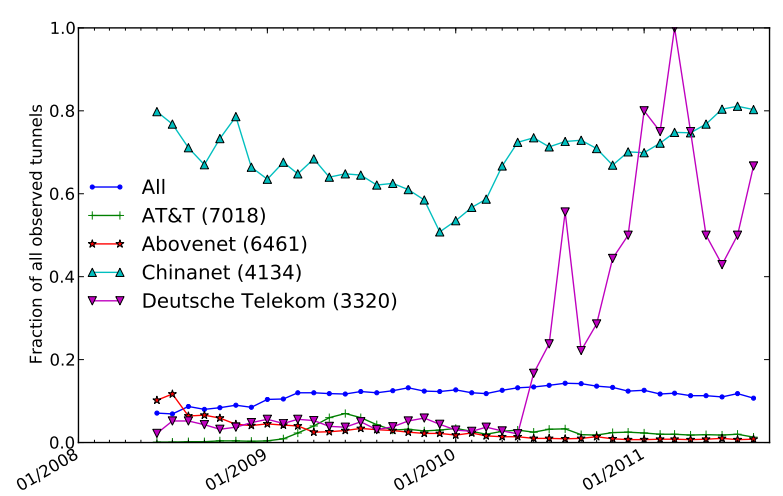

Figure 9: Fraction of tunnels that consist of one hop, over time.

though it is arguable whether this is, in fact, a two-hop tunnel. Given the measurements at hand, we cannot say for certain that an ISP is doing PHP, so the best we can say is that the tunnel is of length 1, and there was one more IP hop in the AS prior to egress.

Next, we examine the distribution of tunnel lengths, both in terms of number of hops, and in latency (milliseconds). Figure 10 shows complementary CDFs for all tunnels, and for three ASes. Note that the $y$-axis is log scale. Lines in each plot correspond to six month periods, starting in January 2009. We choose this form of plot to emphasize the tail of the distribution. We see that the distribution is skewed: for all tunnels, $90 \%$ are 7 hops or fewer, but there are instances of long tunnels, even beyond 15 hops. Plots for individual ASes are somewhat similar to the plot for all ASes. Interestingly, we observe some inflation in path lengths over time for AS6461.

Figure 11 shows similar complementary cumulative distribution function plots of tunnel lengths for all tunnels and the same three ASes, but now considering length in terms of milliseconds. Again, note that the $y$-axis is log scale. Lines in each plot correspond to six month periods, starting in January 2009. We observe that half of all tunnel lengths are approximately zero milliseconds in length, $90 \%$ are 150 milliseconds or less. (Refer to Section 2 for an explanation of why we may see close to zero latency across a tunnel.) We also see that some tunnels exceed 300 milliseconds in length. Tunnel latency distributions for the three ASes differ more obviously than the tunnel hop-length distributions. While the majority of tunnels in ASes 7018 and 6461 are relatively short, 10\% of the tunnels in the most recent six months for AS6453 exceeded 340 milliseconds.

The above analysis considers only the length of inner-most tunnels, i.e., not nested tunnels. In Figure 12 we plot the distribution of lengths of nested tunnels for all ASes for six time periods ( $c f$. the upper left plot in Figure 10. Interestingly, while there is no clear distributional trend in the similar plot in Figure 10, it appears from Figure 12 that nested tunnels appear to be growing longer.

Lastly, we examine the use of multiple depths of label stacks, and the use of different traffic class identifiers. The main uses of label stacking are for supporting VPN services, for more sophisticated traffic engineering, and for LSP protection ("fast reroute") in the event of link or router failure [34]. Since it is highly unlikely that the label stacks we observe are due to VPN services since the Ark nodes are connected to the public Internet, we hypothesize that observed uses of label stacking are due to traffic and network resilience engineering. Figure 13 shows the fraction of tunnels using up to three MPLS labels. Six separate months are shown for all tunnels and for three selected ASes. We see that typically just over $80 \%$ of all tunnels have used a stack of only one label, and virtually no ASes use stacks of three labels. (We observed only a single instance of a label stack of four labels, which was AS3549 in April 2011.) The three ASes shown exhibit much different behavior. While AS7018 used only single-level labels in the earliest measurement period, the majority of its tunnels now employ stacks of two labels. AS2119 was one of the few ASes we observed to employ label stacks of depth 3 (we never observed more than 10 ASes to use 3-deep stacks). We also see that it has very few tunnels that have just a single label on the stack. Lastly, we see that AS19262 has made roughly equal use of label stacks with 1 or 2 labels. In future work we hope to gain insight into the operational practices that lead to these observed behaviors.

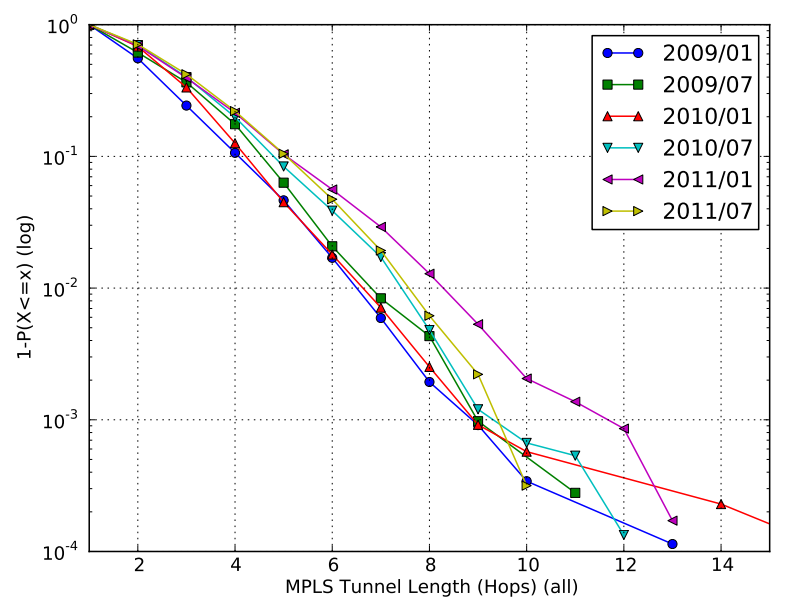

Figure 12: Complementary CDF of the length of all nested tunnels (measured in hops). Note that the $y$-axis is log scale.

The MPLS header contains a 3-bit traffic class field, which can be used by ISPs for implementing different quality of service policies and for prioritizing LSPs. Table 2 shows the fraction of ASes employing different unique traffic class identifiers, over the first month of the past three years. We see that in January 2009, 68\% of all ASes used a single traffic class, $22 \%$ used two classes, and about $10 \%$ used more than two classes. We also see that traffic class identifier usage was roughly the same in January 2010 and 2011.

Table 2: Fraction of ASes employing a given number of traffic class identifiers.

\begin{tabular}{r|ccc}
$\begin{array}{c}\text { Traffic class } \\
\text { labels in use }\end{array}$ & $\mathbf{0 1 / 2 0 0 9}$ & $\mathbf{0 1 / 2 0 1 0}$ & $\mathbf{0 1 / 2 0 1 1}$ \\
\hline 1 & 0.682 & 0.649 & 0.651 \\
2 & 0.223 & 0.224 & 0.283 \\
3 & 0.063 & 0.091 & 0.033 \\
4 & 0.010 & 0.010 & 0.007 \\
5 & 0.006 & 0.006 & 0.004 \\
6 & 0.004 & 0.003 & 0.003 \\
7 & 0.005 & 0.007 & 0.003 \\
8 & 0.006 & 0.006 & 0.010
\end{tabular}

Figure 14 shows cumulative probabilities of using one of the 8 traffic class identifiers across all ASes (left) and in selected ASes (right). We see, similar to Table 2, that the majority of ASes used a single identifier of 0 . However, a number of ASes used a variety of traffic class identifiers. Indeed, we see that AS6799 and AS6834 used the traffic class identifiers with what appears to be almost equal probability. This is surprising, given the fact that the 

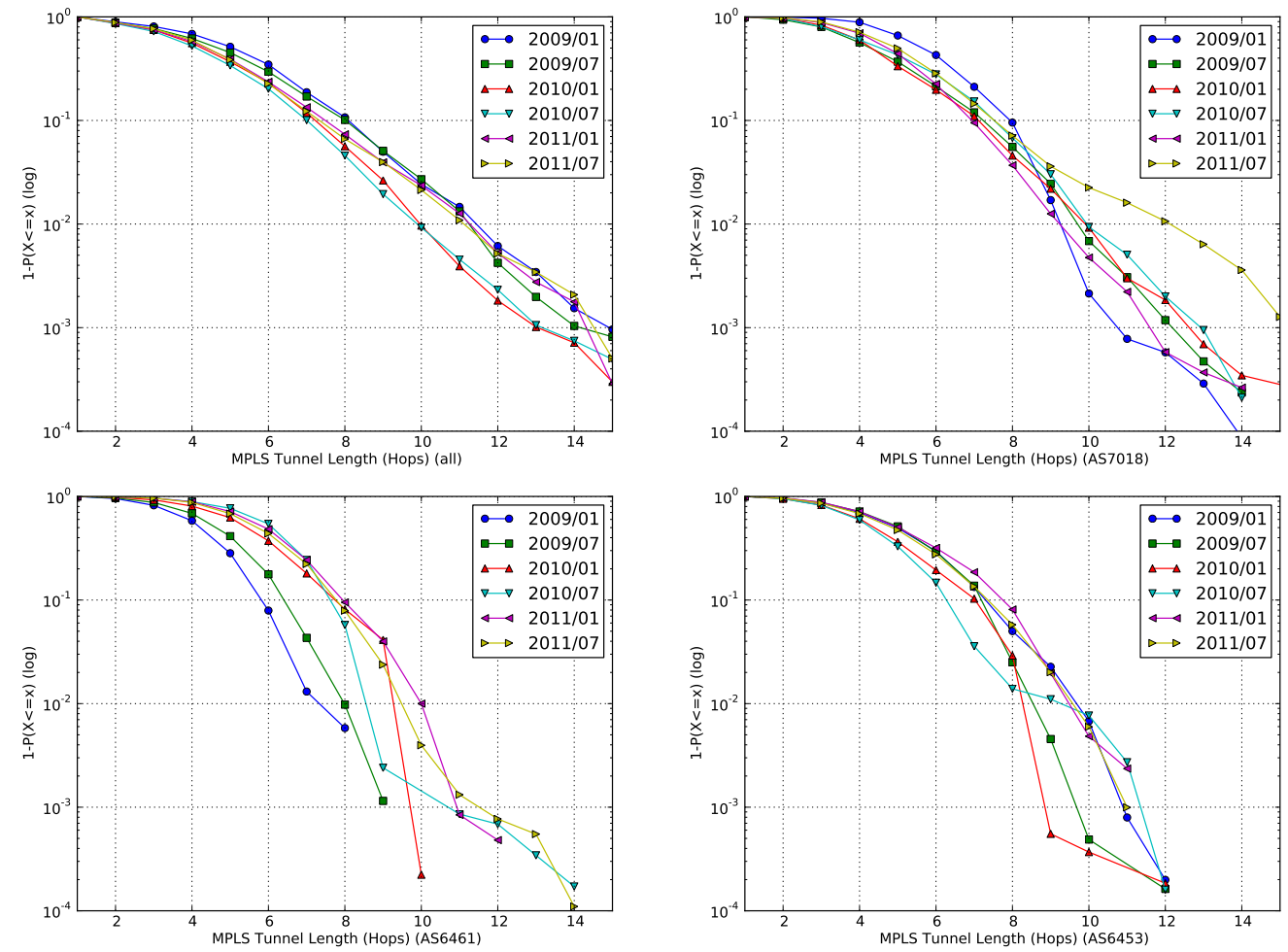

Figure 10: Complementary CDF of length of MPLS tunnels in terms of hops. Note that the y-axis is log scale. Plots shown for all tunnels (upper left) and three selected ASes (7018, 6461, and 6453).
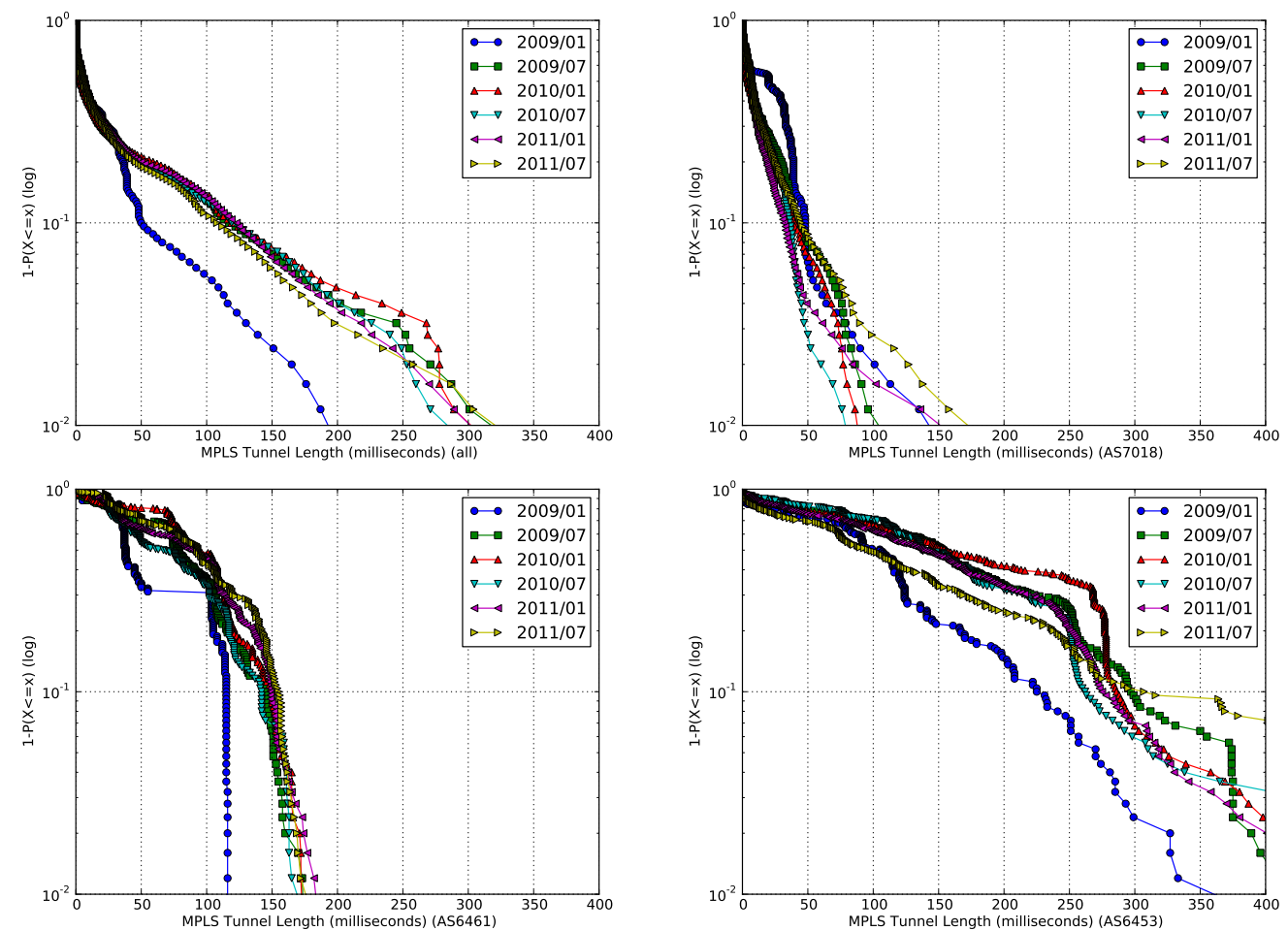

Figure 11: Complementary CDF of length of MPLS tunnels in terms of latency (milliseconds). Note that the y-axis is log scale. Plots shown for all tunnels (upper left) and three selected ASes (7018, 6461, and 6453). 


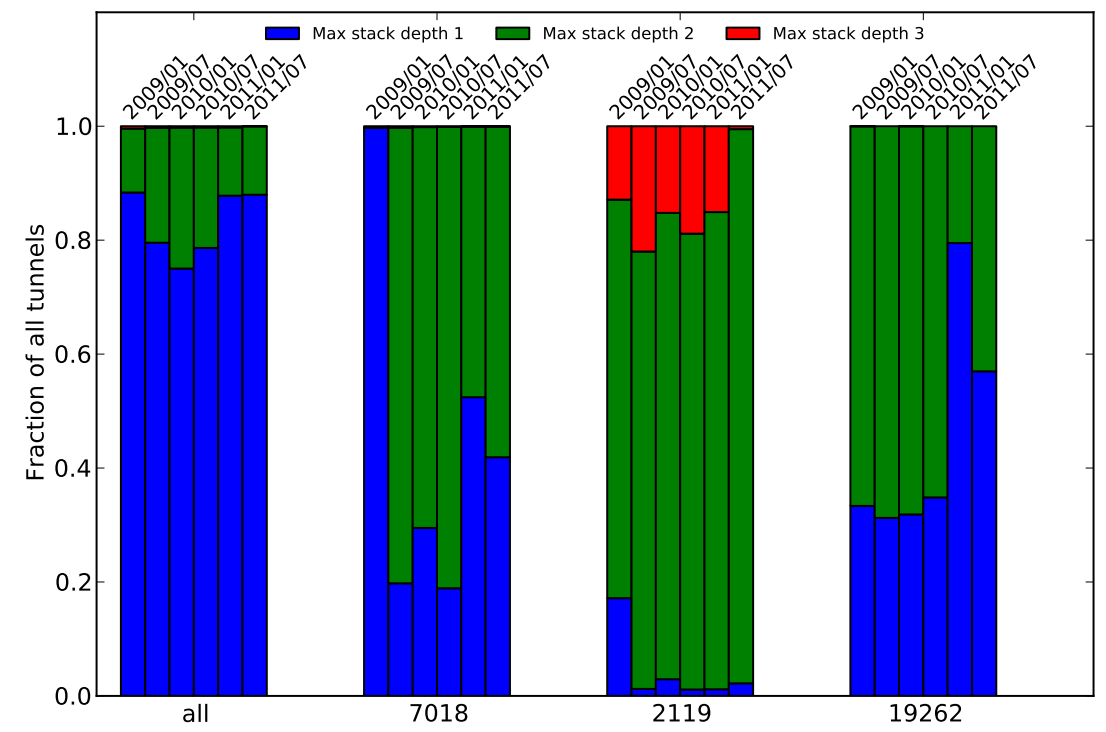

Figure 13: Fraction of tunnels employing different label stack depths, for all tunnels and three selected ASes. Bars for each group are produced from data from one month, as labeled in the plot.

traceroute measurements we analyze are produced using the ICMP-paris method over the entire data collection period, i.e., a single type of source traffic provokes different traffic classifications within the same AS. Taken together, the use of label stacks greater than 1 label and more than 1 traffic class identifier by many ASes suggests that they employ some types of traffic classification and traffic engineering methods. While the details of these methods are not clear, in the future we hope to examine these issues more closely by emitting hop-limited packets using different transport protocols and application payloads along paths that contain MPLS tunnels.

\section{TUNNEL INFERENCE}

Although ICMP extensions enable direct identification of MPLS tunnels in a network, sole reliance on these labels limits the scope of our study. In order to examine the prevalence of MPLS in a wide range of network topologies lacking these MPLS annotations (e.g., the Skitter [13] dataset), this requires the construction of an MPLS inference methodology. We demonstrate how to exploit observable characteristics of MPLS tunnels across various measurement features in order to accurately estimate which paths in a network go through an MPLS tunnel. Note that in this analysis we can only hope to identify tunnels that have been configured in uniform mode, but for which we do not have ICMP extension information to clearly identify the router as being part of an MPLS tunnel.

\subsection{MPLS Tunnel Features}

Consider observable characteristics of a network path which may indicate a particular router interface is in an MPLS tunnel. Assuming traceroute-like probes, we focus on three path properties: latency, hop count, and the IP subnet.

First, consider observations of round-trip time along a traceroute path. As previously described, uniform mode MPLS deployments are configured such that the expiration of a packet within a tunnel causes the packet to be forwarded to the end of the tunnel prior to being routed back to the source host. Intuitively, this results in latency observations for interfaces in the same tun- nel that are all roughly equal. From mining the Ark dataset, we observe the difference in latency between the interfaces under consideration and the next consecutive interfaces in an observed path. Using kernel density estimators [20], we approximate the probability of observed latency given an interface in an MPLS tunnel (i.e., $\widehat{P}_{\text {lat }}^{(k)}(\ell \mid$ MPLS), where $\ell$ is the observed latency between the interface under consideration and the k-hops away interface in the observed path) and the probability of observed latency given that an interface is not in an MPLS tunnel (i.e., $\widehat{P}_{\text {lat }}^{(k)}\left(\ell \mid \operatorname{MPLS}^{C}\right)$ ). In Figure 15 we clearly demonstrate for the April 2011 MPLS-labeled Ark data that MPLS annotated interfaces have different latency characteristics than non-MPLS interfaces. Specifically, for all three figures we find that MPLS interfaces have pairwise latency closer to zero compared with non-MPLS interfaces, which matches our intuition.

We further assume that interfaces in the same MPLS tunnel will be allocated with similar IP addresses, since all the tunnels we observed reside in the same AS. Therefore, two interfaces consecutively found in a path are more likely to be in an MPLS tunnel if they are closer in IP space (e.g., two interfaces in the same $/ 24$ are more likely to be in an MPLS than two interfaces in the same /4). In Figure 16 we plot the MPLS-labeled April 2011 Ark mined kernel density estimates with respect to the observed IP subnet with the k-th consecutive interface in observed paths (i.e., $\widehat{P}_{I P}^{(k)}(s \mid$ MPLS $)$, where $s$ is the observed IP subnet between the interface under consideration and the k-hops away interface in the observed path) and the probability the interface is not in an MPLS tunnel (i.e., $\widehat{P}_{I P}^{(k)}\left(s \mid\right.$ MPLS $\left.^{C}\right)$ ) Again, we find a difference in characteristics between MPLS interfaces and non-MPLS interfaces in this Ark dataset, with MPLS interface having higher IP address subnet compared with non-MPLS interfaces.

Notice that while both of these features show different characteristics for MPLS and non-MPLS interfaces, neither are powerful enough to accurately classify using a single component of information alone. This motivates fusing multiple observed characteristics from the observed path measurement. Specifically, we exploit 

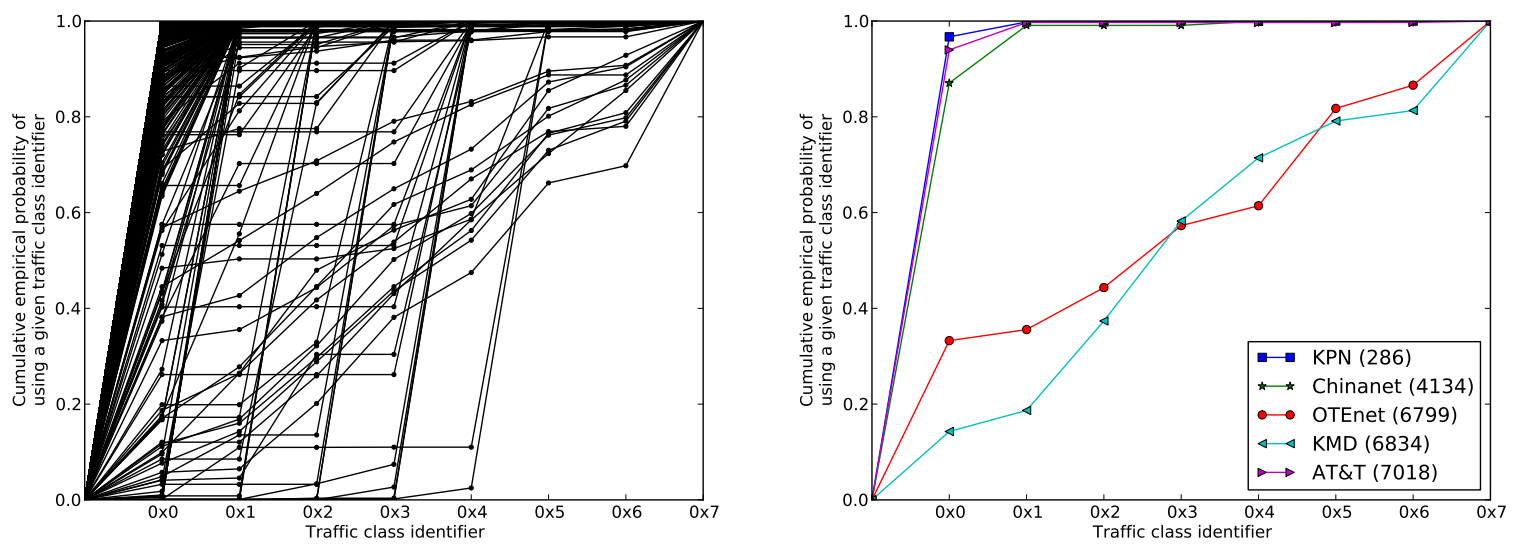

Figure 14: Cumulative empirical probabilities of using different traffic class identifiers for all ASes (left) (each curve corresponds to one AS) and for tunnels in selected ASes (right).
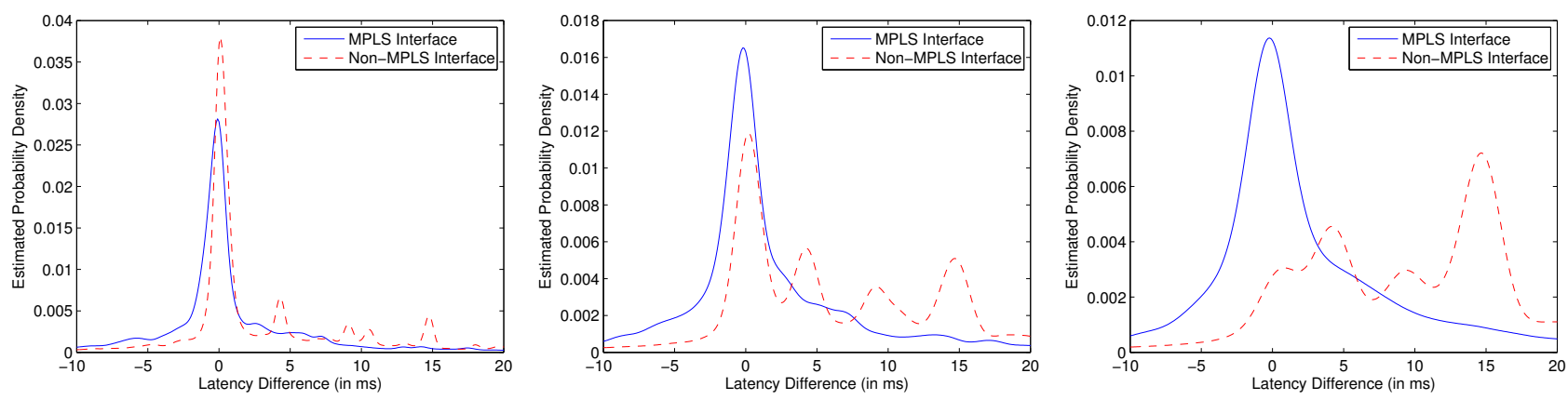

Figure 15: Estimated latency-based distributions between non-MPLS and MPLS interfaces, $\widehat{P}_{\text {lat }}^{(k)}(\ell \mid$ MPLS $)$ and $\widehat{P}_{\text {lat }}^{(k)}\left(\ell \mid\right.$ MPLS $\left.{ }^{C}\right)$, respectively. Where $\ell$ is the latency between interfaces separated by $k$ hops in observed traceroute paths. (Left) interfaces separated by one hop, $k=1$, (Center) interfaces separated by two hops, $k=2$, and (Right) interfaces separated by three hops, $k=3$.

the continuous property of MPLS tunnels in terms of observed hop count. For example, for a path with three interfaces in the same MPLS tunnel, all three of these interfaces must be observed consecutively. This property both reinforces our classification and helps us distinguish between MPLS tunnels and simply an observation of co-located routers in the same PoP along a traceroute path.

\subsubsection{Bayesian Data Fusion Methodology}

We state the probability of an interface $i$ being in an MPLS tunnel given our observed measurements $\left(\mathscr{M}_{i}\right.$, the collection of latencies and IP subnets) for this interface, $P\left(\right.$ MPLS $\left.\mid \mathscr{M}_{i}\right)$, as

$$
\begin{aligned}
P\left(\mathrm{MPLS}_{i}\right) & =P\left(\mathrm{MPLS} \mid \mathscr{M}_{i}\right) \\
P\left(\mathrm{MPLS}_{i}\right) & \propto P\left(\mathscr{M}_{i} \mid \mathrm{MPLS}\right) P(\text { MPLS })
\end{aligned}
$$

Using Bayes Rule (i.e., $P(A \mid B)=\frac{P(B \mid A) P(A)}{P(B)}$ ) and where $\mathscr{M}_{i}$ is the set of observed features from the $K$ interfaces found before and after the interface $i$ in the observed path.

We do not argue that these are the only features that can distinguish MPLS paths; there may indeed be others. This issue is a topic for future investigation.

Unfortunately, as more information is brought to bear on this problem (i.e., the set of features, $\mathscr{M}_{i}$, grows), the higher the dimension of the probability distribution $P\left(\mathscr{M}_{i} \mid\right.$ MPLS $)$ becomes, which results in a computationally intractable problem [20]. In addition, to exploit multiple characteristics from interfaces along the path, it is necessary to fuse together disparate data types (i.e., latency, IP subnet, etc.), for which many off-the-shelf density estimation procedures were not designed. To avoid these limitations, we look to a Naive Bayesian data fusion approach. The Naive Bayesian approach converts the problem from estimating one $M$-dimensional density (which may be computationally intractable) to estimating $M$ one-dimensional densities, such as those estimated in Figure 15 and Figure 16

Using Naive Bayes, the resulting log-likelihood estimated probability for an interface $i$ being in an MPLS tunnel is formulated as:

$$
\begin{aligned}
\log \widehat{P}\left(\mathrm{MPLS}_{i}\right) & =\sum_{k=-K}^{K} \log \widehat{P}_{\text {lat }}^{(k)}\left(\ell_{i, i+k} \mid \mathrm{MPLS}\right) \\
& +\sum_{k=-K}^{K} \log \widehat{P}_{I P}^{(k)}\left(s_{i, i+k} \mid \text { MPLS }\right)
\end{aligned}
$$

While the log-likelihood estimated probability for an interface $i$ not being in an MPLS tunnel is formulated as :

$$
\begin{aligned}
\log \widehat{P}\left(\mathrm{MPLS}_{i}\right) & =\sum_{k=-K}^{K} \log \widehat{P}_{\text {lat }}^{(k)}\left(\ell_{i, i+k} \mid \mathrm{MPLS}^{C}\right) \\
& +\sum_{k=-K}^{K} \log \widehat{P}_{I P}^{(k)}\left(s_{i, i+k} \mid \mathrm{MPLS}^{C}\right)
\end{aligned}
$$



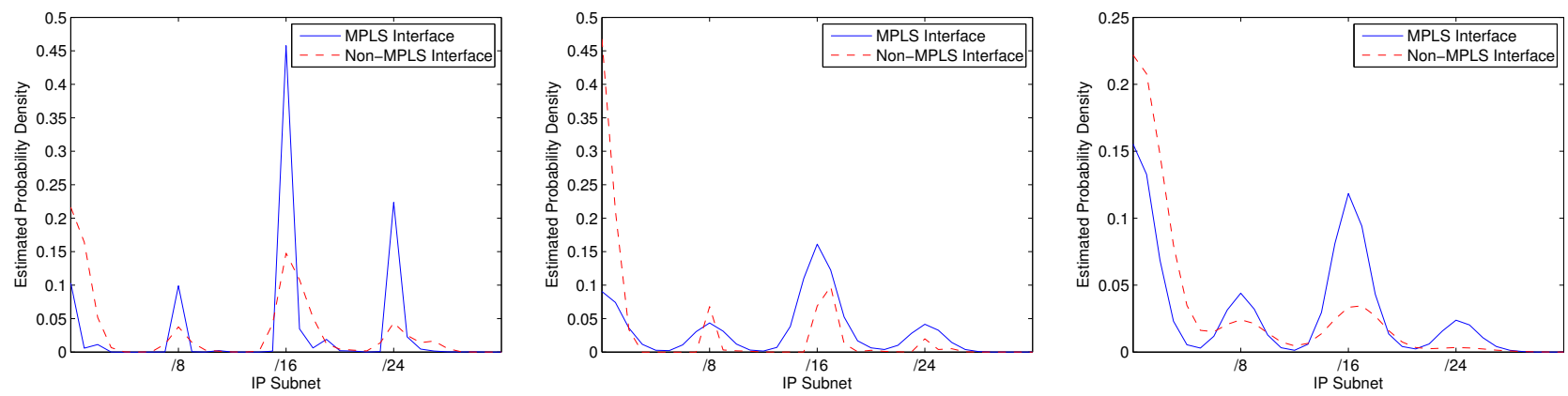

Figure 16: Estimated IP subnet-based distributions between non-MPLS and MPLS interfaces, $\widehat{P}_{I P}^{(k)}\left(s \mid\right.$ MPLS) and $\widehat{P}_{I P}^{(k)}\left(s \mid\right.$ MPLS $\left.{ }^{C}\right)$, respectively. Where $s$ is the IP subnet between interfaces separated by $k$ hops in observed traceroute paths. (Left) interfaces separated by one hop, $k=1$, (Center) interfaces separated by two hops, $k=2$, and (Right) interfaces separated by three hops, $k=3$.

Where $K$ is the number of hops in the observed traceroute path that we examine with respect to the interface $i$.

From this formulation, we see that the computational complexity of this methodology for each interface is only $O(M)$, where $M$ is the number of path features under consideration. On a reasonably equipped host, we find that to resolve the estimated MPLS probability for every interface along a path takes on average 6 milliseconds.

Prior work on using Naive Bayes methodologies on Internet measurements have been explored in the context of IP geolocation in [18]. Our approach differs here through the use of path-based measurements (as opposed to only end-to-end measurements in the prior work) and application (MPLS identification vs. geolocation). We direct the readers to this prior work for a detailed introduction to the Naive Bayes approach.

\subsection{Inference Experimental Results}

We now consider the ability to estimate MPLS tunnels on an Ark dataset from April 2011 containing over 9 million traceroute paths. Due to the use of our learning-based Naive Bayesian inference approach, hold out cross validation [36] is performed to avoid potential bias in our results. This is performed by holding out $5 \%$ of the observed paths (randomly selected) as training data, learning the MPLS characteristics using kernel density estimators, then testing our Bayesian inference method on the remaining $95 \%$ of the observed paths. The detection accuracy results presented are with respect to this held-out test set.

\subsubsection{MPLS Interface Detection}

First, we consider the performance of our methodology with respect to detecting if an interface is in an MPLS tunnel given an observed path containing that interface. Using our Naive Bayesian approach, we fuse together latency, hop count, and IP subnet information to estimate the log-likelihood probability an interface is in an MPLS tunnel. In order to classify our test set of interfaces, we consider a simple thresholding approach between the estimated probability the interface is in an MPLS, against the estimated probability it is not in an MPLS tunnel. The intuition is that the more likely an interface is to be in an MPLS tunnel, the larger this margin will be. Given a set threshold $\lambda$, an interface $i$ is assigned,

$$
i \in\left\{\begin{array}{cl}
\text { MPLS } & : \log \widehat{P}\left(\mathrm{MPLS}_{i}\right)-\log \widehat{P}\left(\mathrm{MPLS}_{i}^{C}\right) \geq \lambda \\
\text { MPLS }^{C} & : \log \widehat{P}\left(\mathrm{MPLS}_{i}\right)-\log \widehat{P}\left(\mathrm{MPLS}_{i}^{C}\right)<\lambda
\end{array}\right.
$$

Using hold-out cross validation on our set of over 9 million paths, the false alarm/detection characteristics for detecting if inter- faces in our test set are in MPLS tunnels can be seen in Figure 17 The figure shows the region operating characteristics (ROC) curve of our classifiers across all feasible values of the threshold $\lambda$ (where each point in the figure represents a different value of $\lambda$ and the associated false alarm and detection rate). These results include our full Naive Bayes data fusion technique (using both latency and IP subnet information), Naive Bayes using only IP subnet information, and Naive Bayes using only latency information. We find that our full technique can detect over 55\% of the MPLS interfaces with only declaring $10 \%$ of the non-MPLS interfaces incorrectly, a significant improvement over using a single characteristic.

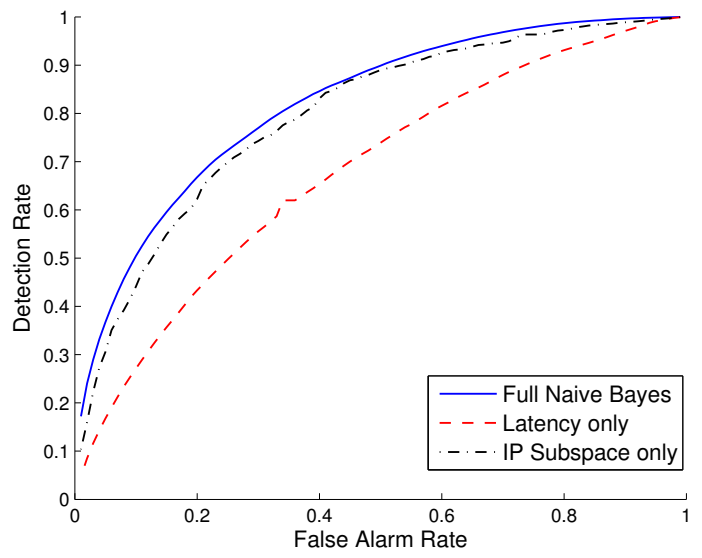

Figure 17: Comparison of the full Naive Bayesian methodology with using only latency or IP subnet to detect MPLS interfaces using traceroute paths from the April 2011 Ark Dataset.

\subsubsection{MPLS Path Detection}

More valuable to a network operator may be knowledge of whether or not a given traceroute path has traversed an MPLS tunnel. In order to classify each observed path as an MPLS path (i.e., a path that crossed an MPLS tunnel) or a non-MPLS path, we consider using the characteristics inferred on each of the interfaces along the observed path. Specifically, we consider aggregating the interface information in the form of the maximum interface log-likelihood probability. Such that, for an observed path 
$\mathbf{p}_{j}=\left\{p_{1}, p_{2}, \ldots, p_{M}\right\}$ we find,

$$
\begin{aligned}
\log \widehat{P}\left(\mathrm{MPLS}_{\mathbf{p}_{j}}\right)=\max _{i=\{1,2, \ldots, M\}}\left(\log \widehat{P}\left(\mathrm{MPLS}_{p_{i}}\right)\right. \\
\left.-\log \widehat{P}\left(\mathrm{MPLS}_{p_{i}}^{C}\right)\right)
\end{aligned}
$$

Again we threshold these margin values against a parameter $\lambda$ to classify each path as MPLS or non-MPLS. As expected, the length of the MPLS tunnel is directly related with the ability to detect if the path does or does not go through an MPLS tunnel. Separating out with respect to MPLS tunnel length, we find the detection characteristics in Figure 18 across the specified April 2011 Ark dataset and all feasible values of $\lambda$.

For MPLS tunnels of length greater than or equal to 4 , we can detect roughly $80 \%$ of the MPLS paths with only a $10 \%$ false alarm rate. This is in contrast to considering all MPLS tunnels, where we only detect $35 \%$ of the tunnels for the same false alarm rate.

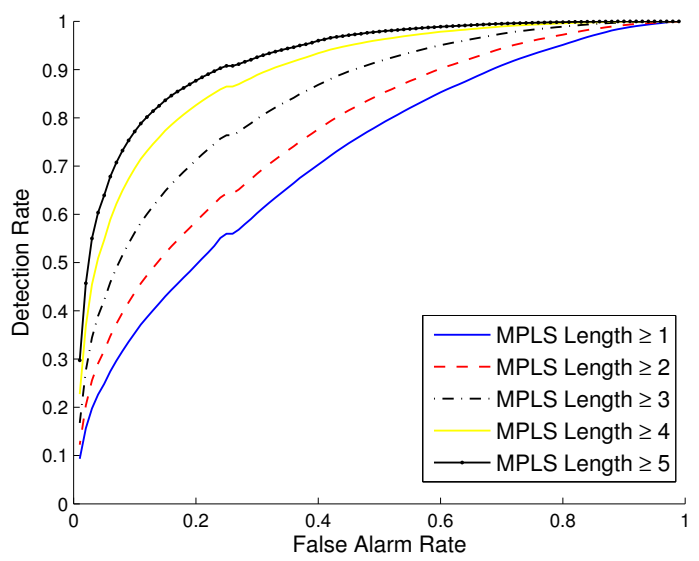

Figure 18: Comparison of the MPLS path detection methodology with respect to the length of the MPLS tunnel using the April 2011 Ark Dataset.

\subsection{Unlabeled MPLS Path Estimation}

Finally, we examine the performance on prior Ark data that does not have MPLS labels. We train our classifier using a subset of labeled June 2008 Ark data, and then test on the prior months (March, April, and May 2008) for which we have no ground truth. We present our results with respect to the four most frequently encountered ASes in our training set. In Table 3, we find the results of our MPLS path detection in terms of the percentage of observed paths for a given autonomous system that we estimate to include an MPLS tunnel. The table shows the percentage of inferred MPLS tunnels as gross characteristics consistent with the labeled June 2008 data. The level of variability in our inference is consistent with the variability that we see in the labeled MPLS data.

While our pre-June 2008 data does not contain uniform-mode MPLS labels, we do have the ability to examine paths that are found in both unlabeled pre-June 2008 data and labeled June 2008 data. Using the June 2008 MPLS labels as ground truth, we can examine performance of our inference methodology on an intersection of these two sets. Figure 19 shows the performance of our inference technique on unlabeled Ark data from March, April, and May 2008. As expected, the classifier performance is more accurate for time frames closer to the labeled set (i.e., May dataset), when compared with the more temporally distant unlabeled set (i.e., March dataset).
Table 3: Comparison of estimated percent of MPLS paths for unlabeled Ark data.

\begin{tabular}{|r||r|r|r|r|r|}
\hline \multicolumn{1}{|c||}{} & \multicolumn{4}{|c|}{ Estimated Paths with Tunnels } & Obs. \\
\hline AS & $03 / 08$ & $04 / 08$ & $05 / 08$ & $06 / 08$ & $06 / 08$ \\
\hline 7018 & $60.7 \%$ & $59.2 \%$ & $76.7 \%$ & $62.5 \%$ & $69.2 \%$ \\
6389 & $54.2 \%$ & $67.5 \%$ & $66.4 \%$ & $69.5 \%$ & $71.0 \%$ \\
4134 & $7.1 \%$ & $8.1 \%$ & $8.5 \%$ & $3.1 \%$ & $0.4 \%$ \\
4230 & $35.5 \%$ & $27.0 \%$ & $29.8 \%$ & $29.4 \%$ & $25.8 \%$ \\
\hline
\end{tabular}

\section{RELATED WORK}

The literature on MPLS largely falls into three categories. The first are studies that describe methods for expanding and enhancing MPLS beyond the original RFCs describing the protocol. Examples include methods for improving reliability and fault tolerance (e.g., [15 25]) and extension for a wide range of label switched paths including photonic networks (i.e., GMPLS [11]) and wireless networks (e.g., [23]). The second category are studies that describe methods for employing MPLS to meet various operational objectives within a given network infrastructure. The most prominent among these are studies that describe a wide variety of traffic engineering methods based on MPLS (e.g., [17, 37|). In the third category are studies describing new routing algorithms that can be used in conjunction with MPLS to establish paths with target characteristics (e.g., [35]).

Details of the MPLS protocol are described in various RFCs which are all linked from the IETF's MPLS working group homepage [3]. The main MPLS architectural reference is RFC 3031 [31], and the most relevant standards document to our study is RFC 4950, which defines the ICMP extensions that enable label stacks to be returned to clients [12]. Beyond RFC documents, Davie and Rekhter wrote a comprehensive textbook reference on MPLS that broadly treats the protocol [16]. There are also numerous online references and notes on practical aspects of MPLS configuration and management (e.g., [34])

We are aware of no prior studies on Internet-wide MPLS deployment characteristics. Perhaps the most relevant empirical studies were by Sherwood et al. in [32 33]. The former study describes a method for measuring router-level topologies that includes the capability to discover MPLS nodes using ICMP extensions. That study provides a small set of measurements on routers that respond with MPLS labels. The latter study also discusses certain aspects of MPLS, again in the context of router-level topology discovery. Our study differs from theirs in its specific focus on MPLS and the longitudinal characterization of its deployment.

\section{SUMMARY AND CONCLUSIONS}

MPLS offers compelling capabilities for traffic engineering, multi-services networks and improved network robustness. In this paper, we describe a longitudinal study of the prevalence and characteristics of MPLS deployments in the open Internet. The goal of our work is to establish a comprehensive baseline for the evolution and current status of MPLS deployments since they have important implications for issues such as quality of service and network neutrality. We use the large traceroute archive from the Ark project as the basis for our work. Over the past 3 years, this data set has included MPLS label stacks, which enable direct evaluation of MPLS tunnels configured in uniform mode. We note again that our direct observations likely underestimate MPLS deployments due to the inability to empirically identify pipe-mode tunnels. 

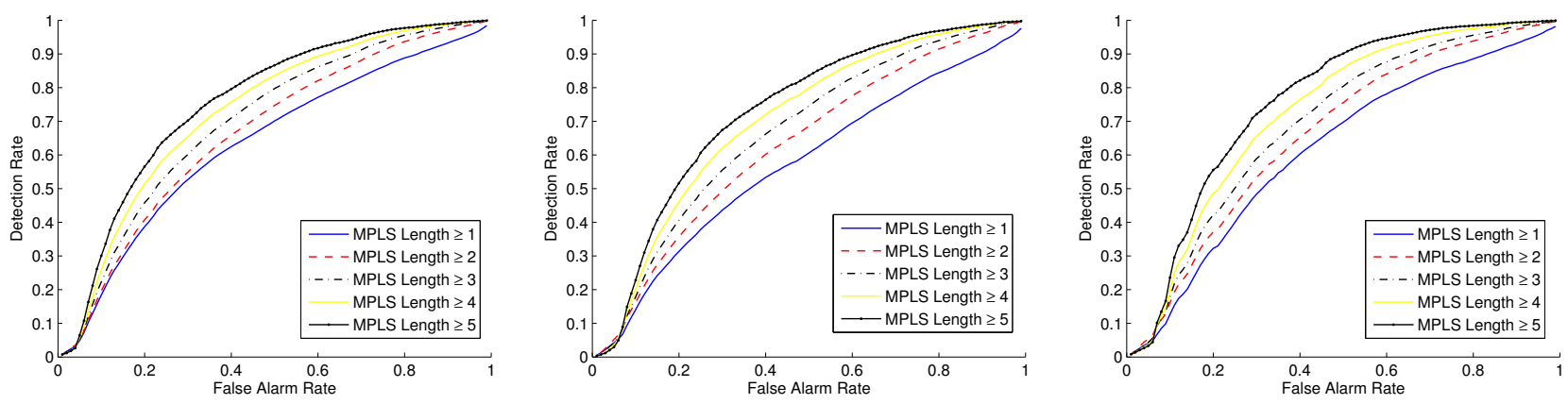

Figure 19: Comparison of the MPLS path detection of unlabeled data using June 2008 labels. (Left) - March 2008 Ark Dataset, (Center) - April 2008 Ark Dataset, (Right) - May 2008 Ark Dataset

The summary findings of our analysis show an increasing trend in MPLS deployments over the past three years and the wide use by tier-1 providers, implying that it is increasingly likely that packets will encounter an MPLS tunnel on end-to-end paths. We also find that tunnels are likely to span the entire edge-to-edge distance of a transit provider, with typical transit times on the order of tens of milliseconds. Lastly, our examination of the use of traffic classifiers indicates that while multiple classes are not uncommon, the diversity of classes has not changed over the past three years.

We develop an MPLS tunnel inference method that is based on the observation that for certain configurations, RTTs for internal hops will all be similar. Our inference method uses Bayesian data fusion to efficiently identify MPLS paths based on RTTs. Using the labeled data, we demonstrate that this method can indeed identify MPLS paths with high accuracy.

In future work, we plan to investigate MPLS deployments in additional traceroute data archives using our Bayesian inference method. A short term target is the Skitter data set, which would give us the opportunity to investigate MPLS deployments over a longer time period. We also plan to expand our survey by conducting more targeted investigations using distributed infrastructures such as Planetlab.

\section{ACKNOWLEDGMENTS}

This work was supported in part by NSF grants CNS-0716460, CNS-0831427 and CNS-0905186, and NSF CAREER award NSF1054985. Any opinions, findings, conclusions or other recommendations expressed in this material are those of the authors and do not necessarily reflect the view of the NSF.

Support for the IPv4 Routed /24 AS Links Dataset is provided by the NSF, the US Department of Homeland Security, the WIDE Project, Cisco Systems, and CAIDA Members. We sincerely thank CAIDA for making these high quality data available to the community.

\section{REFERENCES}

[1] Routeviews Prefix to AS mappings Dataset (pfx2as). http://www.caida.org/data/routing/ routeviews-prefix2as.xml.

[2] Cyclops. http://irl.cs.ucla.edu/topology/data/ Accessed August 2011.

[3] IETF Multiprotocol Label Switching (mpls) Working Group. http: //datatracker. ietf.org/wg/mpls/charter/ Accessed August 2011

[4] Junos OS MPLS Applications Configuration Guide. http: //www.juniper.net/techpubs/en_US/junos11. 1/information-products/topic-collections/ config-guide-mpls-applications/ config-guide-mpls-applications.pdf. Accessed August 2011.

[5] Multiprotocol Label Switching on Cisco Routers. http://www.cisco.com/en/US/docs/ios/12_1t/ 12_1t3/feature/guide/rtr_13t.pdf Accessed August 2011.

[6] Sprint IP/MPLS Network Maps. https://www.sprint.net/network_maps.php Accessed August 2011.

[7] The Internet Topology Zoo. http://www.topology-zoo.org/, Accessed August 2011.

[8] The Traceroute Command in MPLS. http://www.cisco. com/en/US/tech/tk436/tk428/technologies tech_note09186a008020a42a.shtml. Accessed August 2011.

[9] P. Agarwal and B. Akyol. RFC 3443: Time to Live (TTL) Processing in Multi-Protocol Label Switching (MPLS) Networks.

http://www.ietf.org/rfc/rfc3443.txt January 2003.

[10] D. Awduche, J. Malcolm, J. Agogbua, M. O’Dell, and J. McManus. RFC 2702: Requirements for Traffic Engineering Over MPLS.

http://www.ietf.org/rfc/rfc2702.txt September 1999.

[11] L. Berger. RFC 3473: Generalized Multi-Protocol Label Switching (GMPLS) Signaling Resource ReserVation Protocol-Traffiic Engineering (RSVP-TE) Extensions. http://www.ietf.org/rfc/rfc3473.txt January 2003.

[12] R. Bonica, D. Gan, D. Tappan, and C. Pignataro. RFC 4950: ICMP Extensions for Multiprotocol Label Switching. http://www. ietf.org/rfc/rfc4950.txt August 2007.

[13] CAIDA. The Skitter Project. http://www.caida.org/tools/measurement/skitter/, Accessed August 2011. 
[14] R. Callon, P. Doolan, N. Feldman, A. Fredette, and G. Swallow. Draft: A Framework for Multiprotocol Label Switching.http://tools.ietf.org/html/ draft-ietf-mpls-framework-00, May 1997.

[15] H. Chengcheng, V. Sharma, and K. Owens. Building Reliable MPLS Networks Using a Path Protection Mechanism. IEEE Communications Magazine, 40(3), March 2002.

[16] B. Davie and Y. Rekhter. MPLS: Technology and Applications. Morgan Kaufmann, 2000.

[17] A. Elwalid, C. Jin, S. Low, and I. Widjaja. MATE: MPLS Adaptive Traffic Engineering. In Proceedings of IEEE INFOCOM '01, April 2001.

[18] B. Eriksson, P. Barford, J. Sommers, and R. Nowak. A Learning-based Approach for IP Geolocation. In Proceedings of Passive and Active Measurements Conference, April 2010.

[19] F. Le Faucheur et al. RFC 3270: Multi-Protocol Label Switching (MPLS) Support of Differentiated Services. http://www.ietf.org/rfc/rfc3270.txt May 2002.

[20] T. Hastie, R. Tibshirani, and J. Friedman. The Elements of Statistical Learning. Springer, 2001.

[21] Y. Hyun. Personal communication, May 2011.

[22] Y. Hyun, B. Huffaker, D. Andersen, E. Aben, M. Luckie, kc claffy, and C. Shannon. The IPv4 Routed /24 AS Links Dataset: January 2008-August 2011. http://www.caida.org/data/active/ipv4_ routed_topology_aslinks_dataset.xml

[23] H. Kim, K. Wong, W. Chen, and C. Lau. Mobility-aware MPLS in IP-based Wireless Access Networks. In Proceedings of IEEE Globecom '01, November 2001.

[24] K. Kompella and G. Swallow. RFC 4379: Detecting Multi-Protocol Label Switched (MPLS) Data Plane Failures. http://www.ietf.org/rfc/rfc4379.txt February 2006.

[25] S. Lee and M. Gerla. Fault Tolerance and Load Balancing in QoS Provisioning with Multiple MPLS Paths. In Proceedings of the 9th International Workshop on Quality of Service, June 2001.
[26] M. Luckie. Scamper: a Scalable and Extensible Packet Prober for Active Measurement of the Internet. In Proceedings of ACM SIGCOMM Internet Measurement Conference, November 2010.

[27] M. Luckie. Personal communication, May 2011.

[28] M. Luckie, Y. Hyun, and B. Huffaker. Traceroute probe method and forward IP path inference. In Proceedings of ACM SIGCOMM Internet Measurement Conference, pages 311-324, October 2008.

[29] P. Newman, W. Edwards, R. Hinden, E. Hoffman, C. Liaw, T. Lyon, and G. Minshall. RFC 1953: Ipsilon Flow Management Protocol Specification for IP. http://www.ietf.org/rfc/rfc1953.txt May 1996.

[30] Y. Rekhter, B. Davie, D. Katz, E. Rosen, and G. Swallow. RFC 2105: Cisco's Tag Switching Architecture Overview. http://www.ietf.org/rfc/rfc2105.txt February 1997.

[31] E. Rosen, A. Viswanathan, and R. Callon. RFC 3031: Multiprotocol Label Switching Architecture. http://www.ietf.org/rfc/rfc3031.txt January 2001.

[32] R. Sherwood, A. Bender, and N. Spring. Discarte: a disjunctive internet cartographer. In Proceedings of ACM SIGCOMM '08, pages 303-314, August 2008.

[33] R. Sherwood and N. Spring. Touring the Internet in a TCP Sidecar. In Proceedings of ACM SIGCOMM Internet Measurement Conference, pages 339-344, October 2006.

[34] R. Steenbergen. Nanog Tutorial: MPLS for Dummies. http://www.nanog.org/meetings/nanog $49 /$ presentations/Sunday/mpls-nanog49.pdf, June 2010.

[35] B. Wang, S. Xu, and C. Chen. A New Bandwidth Guaranteed Routing Algorithm for MPLS Traffic Engineering. In Proceedings of ICC '02, April 2002.

[36] L. Wasserman. All of nonparametric statistics (springer texts in statistics). Springer, May 2007.

[37] X. Xipeng, A. Hannan, B. Bailey, and L. Ni. Traffic Engineering with MPLS in the Internet. IEEE Network, 14(2), March/April 2000. 